\title{
Defense Responses of Fusarium oxysporum to 2,4-Diacetylphloroglucinol, a Broad-Spectrum Antibiotic Produced by Pseudomonas fluorescens
}

\author{
Alexander Schouten, ${ }^{1}$ Grardy van den Berg, ${ }^{1}$ Véronique Edel-Hermann, ${ }^{2}$ Christian Steinberg, ${ }^{2}$ Nadine \\ Gautheron, ${ }^{2}$ Claude Alabouvette, ${ }^{2}$ C. H. (Ric) de Vos, ${ }^{3}$ Philippe Lemanceau, ${ }^{2}$ and Jos M. Raaijmakers ${ }^{1}$ \\ ${ }^{1}$ Laboratory of Phytopathology, Plant Sciences Department, Wageningen University, P.O. Box 8025, 6700 EE Wageningen, \\ The Netherlands; ' ${ }^{2}$ UMR Microbiologie et Géochimie des Sols, Institut National de la Recherche Agronomique (INRA), 17 \\ rue Sully, 21065 Dijon Cedex, France; ${ }^{3}$ Plant Research International, P.O. Box 16, 6700AA Wageningen, The Netherlands
}

Submitted 20 April 2004. Accepted 5 July 2004.

\begin{abstract}
A collection of 76 plant-pathogenic and 41 saprophytic Fusarium oxysporum strains was screened for sensitivity to 2,4-diacetylphloroglucinol $(2,4-D A P G)$, a broad-spectrum antibiotic produced by multiple strains of antagonistic Pseudomonas fluorescens. Approximately $17 \%$ of the $F$. oxysporum strains were relatively tolerant to high 2,4-DAPG concentrations. Tolerance to 2,4-DAPG did not correlate with the geographic origin of the strains, formae speciales, intergenic spacer (IGS) group, or fusaric acid production levels. Biochemical analysis showed that 18 of 20 tolerant $F$. oxysporum strains were capable of metabolizing 2,4-DAPG. For two tolerant strains, analysis by mass spectrometry indicated that deacetylation of 2,4DAPG to the less fungitoxic derivatives monoacetylphloroglucinol and phloroglucinol is among the initial mechanisms of 2,4-DAPG degradation. Production of fusaric acid, a known inhibitor of 2,4-DAPG biosynthesis in $P$. fluorescens, differed considerably among both 2,4-DAPGsensitive and -tolerant $F$. oxysporum strains, indicating that fusaric acid production may be as important for 2,4DAPG-sensitive as for -tolerant $F$. oxysporum strains. Whether 2,4-DAPG triggers fusaric acid production was studied for six $F$. oxysporum strains; 2,4-DAPG had no significant effect on fusaric acid production in four strains. In two strains, however, sublethal concentrations of 2,4-DAPG either enhanced or significantly decreased fusaric acid production. The implications of 2,4-DAPG degradation, the distribution of this trait within $F$. oxysporum and other plant-pathogenic fungi, and the consequences for the efficacy of biological control are discussed.
\end{abstract}

Additional keyword: detoxification.

The genus Pseudomonas contains not only plant, animal, and human pathogens, but also accommodates species of significant environmental interest, including those that promote plant growth, degrade xenobiotics, or control plant diseases. Numerous antibiotics have been isolated from various Pseudomonas strains, including phenazines, lipodepsipeptides, and phloroglucinols (Chin-A-Woeng et al. 2003; de Souza et al. 2003b,c; Nielsen et al. 2002; Raaijmakers et al. 2002). Phloro-

Corresponding author: A. Schouten; Telephone: +31 317 483131; Fax: +31317 483412; E-mail: sander.schouten@wur.nl glucinols are phenolic compounds produced by Pseudomonas spp., but also by plants and algae (de Souza et al. 2003a). More than 60 phloroglucinol derivatives have been described and were reported to have antiviral, antibacterial, antifungal, antihelminthic, phytotoxic, antitumor, and plant growth-regulating activities (de Souza et al. 2003a). The broad-spectrum activity of 2,4-diacetylphloroglucinol (2,4-DAPG) produced by Pseudomonas fluorescens strains recently has drawn attention in the medical area due to its bacteriolytic activity against multidrug-resistant Staphylococcus aureus (Kamei and Isnansetyo 2003). Over the past decade, 2,4-DAPG has been shown to play a key role in the biological control of various plant pathogens, including Fusarium oxysporum (Duffy et al. 2004; Duffy and Défago 1997; Keel et al. 1996; Thomashow and Weller 1996).

F. oxysporum contains opportunistic human pathogens, antagonists, and plant pathogens (Summerbell and Schroers 2002). Plant-pathogenic F. oxysporum causes substantial yield losses in many economically important crops and is considered to be the most important soil borne Fusarium spp. F. oxysporum produces an array of toxins (Chelkowski 1989), including mycotoxins, that have adverse effects on human and animal health (Gupta et al. 2000; Joffe 1986). During the saprophytic and parasitic stages of its life cycle, $F$. oxysporum is exposed to multiple toxic compounds, including antifungal metabolites produced by microorganisms that reside in the same microsites (Chin-AWoeng et al. 2003; Lagopodi et al. 2002). Evidently, F. oxysporum must be able to tolerate or combat these toxic metabolites to propagate, colonize, and successfully infect plants.

Various mechanisms that enable microorganisms to resist toxic compounds have been described in the areas of medical microbiology, bioremediation, and plant-pathogen interactions, and include enzymatic degradation or inactivation of antibiotic compounds, alteration of the target sites, and active efflux (Alexander et al. 1999; De Waard 1997; Fleissner et al. 2002; Morrissey and Osbourn 1999; Schoonbeek et al. 2002; Steffens et al. 1996; VanEtten et al. 2001). Relatively little is known about the role of these mechanisms in fungal defense against microbial antagonism. Studies have shown that, within several fungal populations, there is variation in sensitivity to antifungal metabolites produced by antagonistic bacteria (Duffy et al. 2003). For example, various isolates of the take-all fungus differ considerably in their sensitivity toward 2,4-DAPG and phenazines produced by Pseudomonas spp. (Mazzola et al. 1995). With respect to defense responses 
in $F$. oxysporum to antibiotics produced by antagonistic Pseudomonas spp. Duffy and Défago (1997) elegantly demonstrated that fusaric acid, a pyridine-carboxylic acid, acts as a signal that specifically represses 2,4-DAPG biosynthesis in the antagonistic strain $P$. fluorescens CHA0. Fusaric acid was shown to act as a repressor of phlA expression both in vitro and in situ (Notz et al. 2002; Schnider-Keel et al. 2000).
Blocking fusaric acid production by the pathogen via zinc amendment relieved phlA repression and improved the biocontrol activity of strain CHA0 (Duffy and Défago 1997). Recent studies have further shown that certain 2,4-DAPGproducing Pseudomonas strains are relatively insensitive to fusaric acid as a negative signal in 2,4-DAPG synthesis (Duffy et al. 2003, 2004).

Table 1. Strains of Fusarium oxysporum analyzed in this study ${ }^{\mathrm{a}}$

\begin{tabular}{|c|c|c|c|c|c|c|c|}
\hline Strain & Formae speciales & Isolated from & Country & Year & IGS type & $\begin{array}{l}\text { 2,4-DAPG } \\
\text { tolerance }^{c}\end{array}$ & Source, reference ${ }^{d}$ \\
\hline Foln 3 & lini & Flax & France & 1981 & 12 & - & FPFS \\
\hline Fol8 & lycopersisci & Tomato & France & 1971 & 23 & - & FPFS \\
\hline Fol15 & lycopersisci & Tomato & Tunisia & 1968 & 25 & - & FPFS \\
\hline Fol24 & lycopersisci & Tomato & France & 1977 & 23 & - & FPFS \\
\hline Fol27 & lycopersisci & Soil & France & 1980 & 25 & - & FPFS \\
\hline Fol28 & lycopersisci & Soil & France & 1980 & 25 & - & FPFS \\
\hline Fol29 & lycopersisci & Tomato & France & 1981 & 25 & - & FPFS \\
\hline Fol32 & lycopersisci & Tomato & France & 1985 & 23 & - & FPFS \\
\hline Fol33 & lycopersisci & Tomato & United States & 1987 & 25 & - & FPFS \\
\hline Fol34 & lycopersisci & Tomato & United States & 1987 & 25 & - & FPFS \\
\hline Fol35 & lycopersisci & Tomato & Japan & NA & ND & - & FPFS, 880621a-1 (T. Arie) \\
\hline Fom3 & melonis & Melon & France & 1962 & ND & - & FPFS \\
\hline Fom7 & melonis & Melon & France & 1964 & ND & - & FPFS \\
\hline Fom 15 & melonis & Melon & France & 1965 & ND & - & FPFS \\
\hline Fom 20 & melonis & Melon & France & 1965 & ND & - & FPFS \\
\hline Fom 24 & melonis & Melon & France & 1968 & 21 & - & FPFS \\
\hline Fom 28 & melonis & Melon & Netherlands & 1970 & ND & + & FPFS \\
\hline Fom 31 & melonis & Melon & France & 1970 & ND & - & FPFS \\
\hline Fom 32 & melonis & Melon & France & 1970 & ND & - & FPFS \\
\hline Fom 35 & melonis & Melon & France & 1985 & ND & - & FPFS \\
\hline Fom 37 & melonis & Melon & France & 1985 & ND & + & FPFS \\
\hline Fom 38 & melonis & Melon & United States & NA & ND & ++ & FPFS, MC6-8S (T. R. Gordon) \\
\hline Fom39 & melonis & Melon & United States & NA & ND & - & FPFS, KNH (T. R. Gordon) \\
\hline Fom 117 & melonis & Melon & Turkey & 1962 & ND & - & FPFS \\
\hline Fom 1127 & melonis & Melon & United States & 1987 & ND & ++ & FPFS, Fom 1127 (T. R. Gordon) \\
\hline Focub1 & cubense & Banana & Taiwan & NA & ND & ++ & FPFS \\
\hline Focub2 & cubense & Banana & Taiwan & NA & ND & ++ & FPFS \\
\hline Focub3 & cubense & Banana & Taiwan & NA & ND & - & FPFS \\
\hline Focub6 & cubense & Banana & Taiwan & NA & ND & - & FPFS \\
\hline Focub7 & cubense & Banana & Taiwan & NA & ND & - & FPFS \\
\hline Focub9 & cubense & Banana & Taiwan & NA & ND & - & FPFS \\
\hline Focub13 & cubense & Banana & Taiwan & NA & ND & ++ & FPFS \\
\hline Focub16 & cubense & Banana & Taiwan & NA & ND & - & FPFS \\
\hline Fob2 & basilicum & Basilicum & France & 1994 & ND & - & FPFS \\
\hline Fob3 & basilicum & Basilicum & Italy & NA & ND & + & FPFS \\
\hline Fod 1 & dianthi & Soil & France & 1987 & ND & + & FPFS \\
\hline Fod 2 & dianthi & Carnation & Netherlands & NA & 50 & - & FPFS, WCS816 (R. P. Baayen) \\
\hline Fod3 & dianthi & Carnation & Argentina & NA & ND & - & FPFS, CBS214.49 \\
\hline Fod4 & dianthi & Carnation & Argentina & NA & ND & - & FPFS \\
\hline Fod8 & dianthi & Carnation & Netherlands & NA & 64 & - & FPFS, NAKS3 (R. P. Baayen) \\
\hline For1 & raphani & Radish & Netherlands & NA & ND & - & FPFS, WCS600 \\
\hline For2 & raphani & Radish & Unknown & NA & ND & - & FPFS \\
\hline For3 & raphani & Radish & Germany & NA & ND & - & FPFS \\
\hline For4 & raphani & Radish & France & NA & ND & - & FPFS \\
\hline Foci1 & ciceri & Chick pea & Spain & 1990 & ND & - & FPFS \\
\hline Foci2 & ciceri & Chick pea & Tunisia & 1991 & ND & - & FPFS \\
\hline Foci4 & ciceri & Chick pea & Spain & 1980 & ND & - & FPFS \\
\hline Focy1 & cyclamini & Cyclamen & France & 1994 & ND & - & FPFS \\
\hline Focy 2 & cyclamini & Cyclamen & France & 1994 & ND & - & FPFS \\
\hline Foe1 & elaidis & Oil palm & Ivory Coast & NA & ND & - & FPFS \\
\hline Foa1 & albedinis & Date palm & Morocco & 1966 & ND & - & FPFS \\
\hline Foa2 & albedinis & Date palm & Algeria & 1972 & ND & + & FPFS \\
\hline Foa3 & albedinis & Date palm & Algeria & 1973 & ND & - & FPFS \\
\hline \multirow[t]{2}{*}{ Foa4 } & albedinis & Date palm & Morocco & 1984 & ND & - & FPFS \\
\hline & & & & & & & (continued on next page) \\
\hline
\end{tabular}

a NA = not available and ND = not determined.

${ }^{b}$ Restriction patterns revealed with seven restriction enzymes for each intergenic spacer (IGS) type were described previously (Edel et al. 1995, 1997, 2001; Lori et al. 2004).

c Tolerant strains were divided into three categories depending on their radial growth with 2,4-diacetylphloroglucinol (2,4-DAPG) at 400 $\mu$ g/ml: strains for which the radial growth was reduced by more than $90 \%(+)$, strains for which the radial growth was reduced by 70 to $90 \%(++)$, and strains for which the radial growth was reduced by 50 to $70 \%$ (+++).

${ }^{\mathrm{d}}$ FPFS: Collection of Flore Pathogène et Faune du Sol, UMR Microbiologie et Géochimie des Sols (MGS), Institut National de la Recherche Agronomique (INRA), Dijon, France. WU-Phyto: Department of Phytopathology, Wageningen University, Wageningen, The Netherlands. 
In this study, a large collection of $F$. oxysporum strains obtained from various host plants, soil, and geographical locations and representing multiple intergenic spacer (IGS) groups was screened for sensitivity toward 2,4-DAPG. Strains tolerant to 2,4-DAPG were further analyzed at the biochemical level with respect to the nature of their defense mechanism against 2,4-DAPG. Finally, the effect of 2,4-DAPG on fusaric acid production in several $F$. oxysporum strains was monitored.

\section{RESULTS}

Genotypic diversity of $\boldsymbol{F}$. oxysporum.

A subsample of 32 pathogenic strains was characterized by RFLP analysis of the ribosomal IGS. The 32 pathogenic strains were assigned to eight IGS types (Table 1). The 40 nonpathogenic strains were representative of 17 IGS types (Edel et al. 1995, 1997, 2001). Two identical IGS types were found

Table 1. (continued from preceding page)

\begin{tabular}{|c|c|c|c|c|c|c|c|}
\hline Strain & Formae speciales & Isolated from & Country & Year & IGS type ${ }^{b}$ & $\begin{array}{l}\text { 2,4-DAPG } \\
\text { tolerance }^{c}\end{array}$ & Source, reference ${ }^{d}$ \\
\hline Foa5 & albedinis & Date palm & Morocco & 1985 & ND & - & FPFS \\
\hline Foap2 & asparagi & Asparagus & Netherlands & 1990 & ND & + & FPFS, CWB6 (Wim Blok) \\
\hline Forl1 & radicis lycopersici & Tomato & France & 1991 & 32 & - & FPFS \\
\hline Forl2 & radicis lycopersici & Tomato & France & 1991 & 25 & - & FPFS \\
\hline Forl6 & radicis lycopersici & Tomato & France & 1993 & 25 & - & FPFS \\
\hline Forl7 & radicis lycopersici & Tomato & France & 1994 & 25 & - & FPFS \\
\hline Forl8 & radicis lycopersici & Tomato & France & 1995 & 32 & - & FPFS \\
\hline Forl9 & radicis lycopersici & Tomato & France & 1995 & 32 & - & FPFS \\
\hline Forl10 & radicis lycopersici & Tomato & France & 1995 & 32 & - & FPFS \\
\hline Forl12 & radicis lycopersici & Tomato & Morocco & 1995 & 25 & - & FPFS \\
\hline Forl14 & radicis lycopersici & Tomato & France & 1992 & 23 & - & FPFS \\
\hline Forl15 & radicis lycopersici & Tomato & France & 1994 & 3 & - & FPFS \\
\hline Forl19 & radicis lycopersici & Tomato & France & 1985 & 25 & - & FPFS \\
\hline Forl22 & radicis lycopersici & Tomato & France & 1985 & 25 & - & FPFS \\
\hline Forl23 & radicis lycopersici & Tomato & France & 1986 & 25 & - & FPFS \\
\hline Forl25 & radicis lycopersici & Tomato & France & 1986 & 25 & - & FPFS \\
\hline Forl31 & radicis lycopersici & Tomato & France & 1997 & 32 & - & FPFS \\
\hline Forl43 & radicis lycopersici & Tomato & France & 1998 & 32 & - & FPFS \\
\hline Forl50 & radicis lycopersici & Tomato & France & 1998 & 25 & - & FPFS \\
\hline Forl60 & radicis lycopersici & Tomato & Canada & NA & 23 & - & FPFS \\
\hline Forl68 & radicis lycopersici & Tomato & France & 1998 & 32 & - & FPFS \\
\hline Forl70 & radicis lycopersici & Tomato & Japan & NA & ND & - & FPFS, SUF959 (T. Arie) \\
\hline Fo47 & $\ldots$ & Soil & France & 1978 & 22 & - & FPFS, Edel et al. 2001 \\
\hline Fo5A4 & $\ldots$ & Soil & France & 1984 & 5 & - & FPFS, Edel et al. 2001 \\
\hline 91002 & $\ldots$ & Soil & France & 1991 & 1 & - & FPFS, Edel et al. 1997 \\
\hline 91004 & $\ldots$ & Soil & France & 1991 & 4 & - & FPFS, Edel et al. 1997 \\
\hline 91014 & $\ldots$ & Soil & France & 1991 & 14 & + & FPFS, Edel et al. 1997 \\
\hline 91015 & $\ldots$ & Soil & France & 1991 & 11 & ++ & FPFS, Edel et al. 1997 \\
\hline 91069 & $\ldots$ & Soil & France & 1991 & 3 & ++ & FPFS, Edel et al. 1997 \\
\hline 91121 & $\ldots$ & Soil & France & 1991 & 2 & - & FPFS, Edel et al. 1997 \\
\hline 91142 & $\ldots$ & Soil & France & 1991 & 8 & - & FPFS, Edel et al. 1997 \\
\hline 91248 & $\ldots$ & Soil & France & 1991 & 9 & - & FPFS, Edel et al. 1997 \\
\hline 91426 & $\ldots$ & Soil & France & 1991 & 15 & +++ & FPFS, Edel et al. 1997 \\
\hline 92008 & $\ldots$ & Soil & France & 1992 & 16 & - & FPFS, Edel et al. 1997 \\
\hline 92015 & $\ldots$ & Soil & France & 1992 & 7 & - & FPFS, Edel et al. 1997 \\
\hline 92018 & $\ldots$ & Soil & France & 1992 & 10 & - & FPFS, Edel et al. 1997 \\
\hline $91 \mathrm{~B} 2$ & $\ldots$ & Wheat & France & 1991 & 4 & - & FPFS, Edel et al. 1997 \\
\hline $91 \mathrm{~B} 3$ & $\ldots$ & Wheat & France & 1991 & 4 & - & FPFS, Edel et al. 1997 \\
\hline $91 \mathrm{~B} 9$ & $\ldots$ & Wheat & France & 1991 & 4 & - & FPFS, Edel et al. 1997 \\
\hline $91 \mathrm{~B} 12$ & $\ldots$ & Wheat & France & 1991 & 4 & - & FPFS, Edel et al. 1997 \\
\hline $91 \mathrm{~B} 13$ & $\ldots$ & Wheat & France & 1991 & 4 & - & FPFS, Edel et al. 1997 \\
\hline 91B18 & $\ldots$ & Wheat & France & 1991 & 4 & - & FPFS, Edel et al. 1997 \\
\hline $91 \mathrm{~B} 50$ & $\ldots$ & Wheat & France & 1991 & 4 & ++ & FPFS, Edel et al. 1997 \\
\hline 91B51 & $\ldots$ & Wheat & France & 1991 & 4 & - & FPFS, Edel et al. 1997 \\
\hline $91 \mathrm{~B} 52$ & $\ldots$ & Wheat & France & 1991 & 4 & - & FPFS, Edel et al. 1997 \\
\hline $91 \mathrm{~B} 53$ & $\ldots$ & Wheat & France & 1991 & 4 & - & FPFS, Edel et al. 1997 \\
\hline $91 \mathrm{~B} 54$ & $\ldots$ & Wheat & France & 1991 & 4 & - & FPFS, Edel et al. 1997 \\
\hline $91 \mathrm{~B} 56$ & $\ldots$ & Wheat & France & 1991 & 4 & - & FPFS, Edel et al. 1997 \\
\hline $92 \mathrm{~B} 11$ & $\ldots$ & Wheat & France & 1992 & 4 & - & FPFS, Edel et al. 1997 \\
\hline $92 \mathrm{~T} 1$ & $\ldots$ & Wheat & France & 1992 & 4 & - & FPFS, Edel et al. 1997 \\
\hline $92 \mathrm{~T} 6$ & $\ldots$ & Wheat & France & 1992 & 4 & - & FPFS, Edel et al. 1997 \\
\hline $92 \mathrm{~T} 11$ & $\ldots$ & Tomato & France & 1992 & 11 & + & FPFS, Edel et al. 1997 \\
\hline $92 \mathrm{~T} 60$ & $\ldots$ & Tomato & France & 1992 & 11 & - & FPFS, Edel et al. 1997 \\
\hline Carq1 & $\ldots$ & Soil & France & 1996 & 5 & - & FPFS, Edel et al. 2001 \\
\hline Carq2 & $\ldots$ & Soil & France & 1996 & 3 & - & FPFS, Edel et al. 2001 \\
\hline Carq6 & $\ldots$ & Soil & France & 1996 & 37 & ++ & FPFS, Edel et al. 2001 \\
\hline Carq38 & $\ldots$ & Soil & France & 1996 & 14 & - & FPFS, Edel et al. 2001 \\
\hline Carq290 & $\ldots$ & Soil & France & 1996 & 23 & - & FPFS, Edel et al. 2001 \\
\hline Chat3 & $\ldots$ & Soil & France & 1996 & 5 & - & FPFS, Edel et al. 2001 \\
\hline Chat7 & $\ldots$ & Soil & France & 1996 & 3 & + & FPFS, Edel et al. 2001 \\
\hline Chat59 & $\ldots$ & Soil & France & 1996 & 20 & - & FPFS, Edel et al. 2001 \\
\hline Chat 214 & $\ldots$ & Soil & France & 1996 & 23 & - & FPFS, Edel et al. 2001 \\
\hline $\mathrm{R} 23$ & $\ldots$ & Wheat rhizosphere & Netherlands & 2002 & ND & +++ & WU-Phyto \\
\hline
\end{tabular}


among both pathogenic and nonpathogenic $F$. oxysporum strains; therefore, a total of 23 IGS types were found. The genetic relationships between the IGS types detected among the strains analyzed are illustrated in Figure 1.

\section{Sensitivity of $\boldsymbol{F}$. oxysporum for 2,4-DAPG.}

Sensitivity of the $117 F$. oxysporum strains for 2,4-DAPG was tested on one-fifth-strength PDA plates supplemented with 2,4-DAPG at concentrations ranging from 0 to $400 \mu \mathrm{g} / \mathrm{ml}$. The radial growth was measured after 5 days of incubation at $25^{\circ} \mathrm{C}$. Mycelial growth of many strains was completely inhibited at 2,4-DAPG concentrations of 25 and $50 \mu \mathrm{g} / \mathrm{ml}$. These strains were considered 2,4-DAPG sensitive (Table 1; Fig. 2). Eleven pathogenic and nine nonpathogenic strains were able to grow at 2,4-DAPG concentrations of up to $400 \mu \mathrm{g} / \mathrm{ml}$, although growth for most strains was reduced compared with the control (Fig. 2). These strains were considered 2,4-DAPG tolerant and included pathogenic F. oxysporum strains Fom28,
Fom37, Fom38, Fom1127, Focub1, Focub2, Focub3, Focub13, Fob3, Fod1, Foa2, and Foap2, and nonpathogenic F. oxysporum strains 91014, 91015, 91069, 91426, 91B50, 92T11, Carq6, Chat7, and R23. On average, 2,4-DAPG-tolerant strains accounted for approximately $17 \%$ of the total number of strains tested.

Tolerant $F$. oxysporum strains degrade 2,4-DAPG.

At a 2,4-DAPG concentration of $400 \mu \mathrm{g} / \mathrm{ml}$, the antibiotic partly precipitates in the agar medium, resulting in opaque plates. Visual and microscopic inspection of plates amended with 2,4-DAPG at $400 \mu \mathrm{g} / \mathrm{ml}$ revealed that all but two tolerant F. oxysporum strains (91015 and 91B50) showed a clear zone (halo) underneath and, in many cases, up to $3 \mathrm{~mm}$ in front of the growing mycelium (Fig. 3). These results indicated that the 2,4-DAPG crystals had disappeared. To determine the fate of 2,4-DAPG, an extraction was performed on three different zones followed by high-performance liquid chromatography

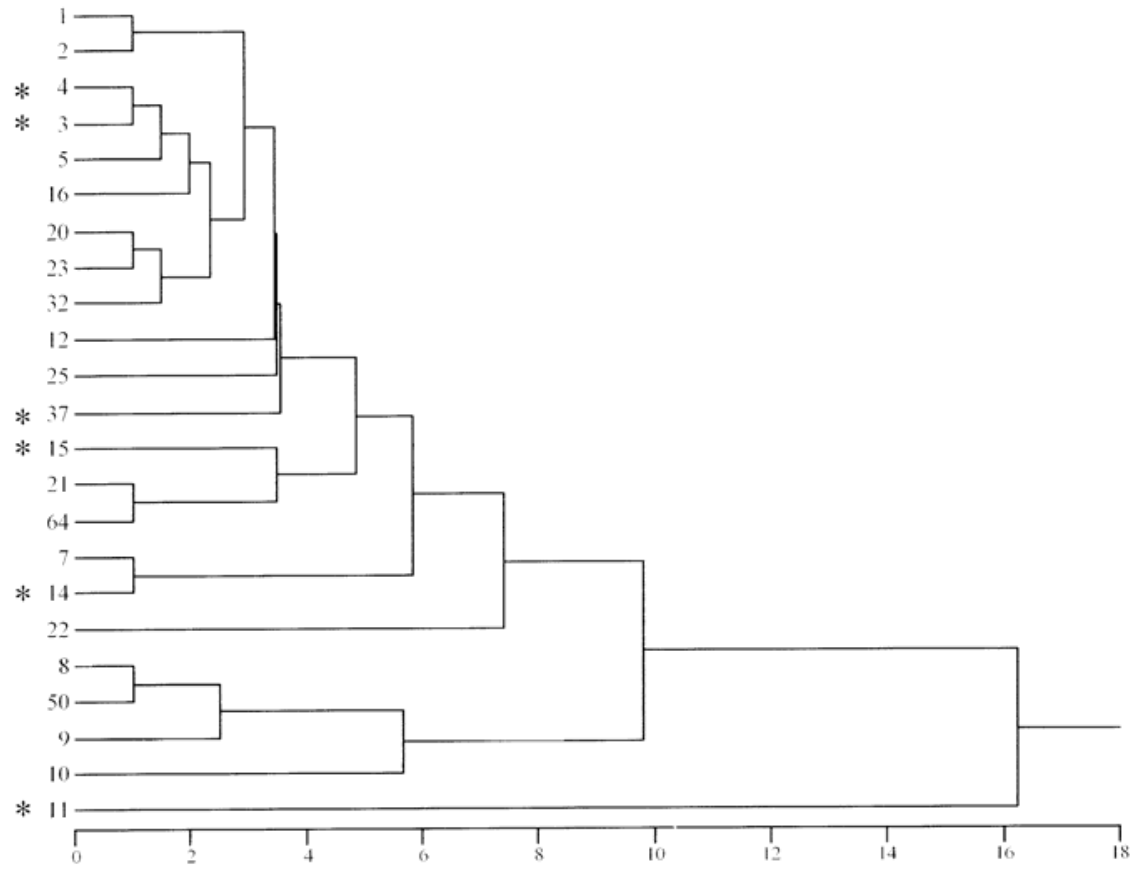

Fig. 1. Dendrogram showing the restriction site differences in the intergenic spacer (IGS) region of Fusarium oxysporum strains representing 23 IGS types. The IGS types marked by an asterisk include 2,4-diacetylphloroglucinol-tolerant strains of $F$. oxysporum. The scale indicates the number of restriction site differences.

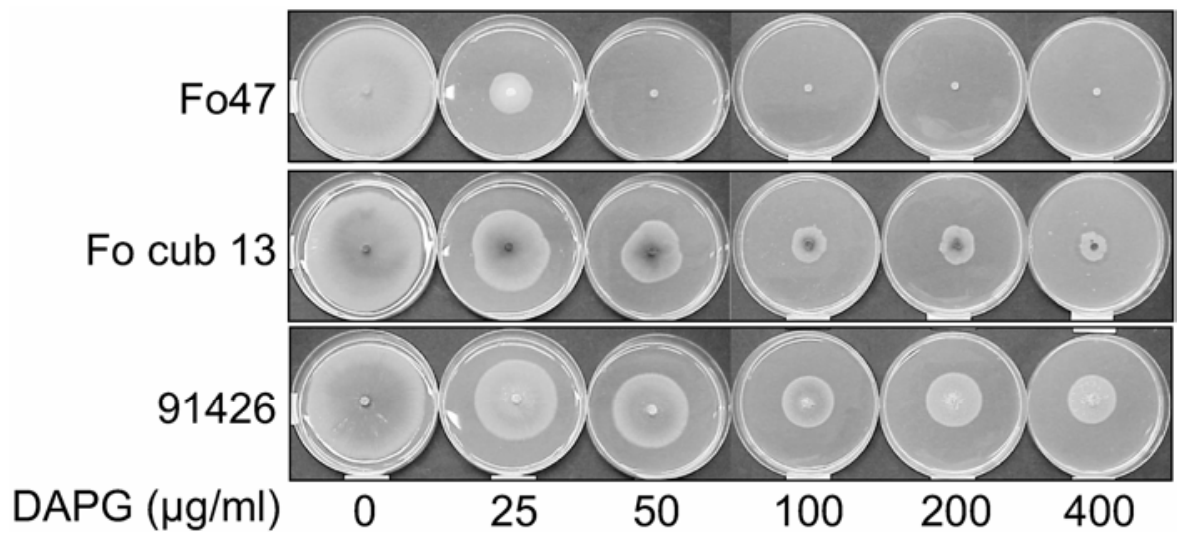

Fig. 2. In vitro assay to determine the sensitivity of the Fusarium oxysporum strains for different concentrations of 2,4-diacetylphloroglucinol (2,4-DAPG) present in one-fifth-strength potato dextrose agar plates. Displayed are three different strains. Fo47 is considered a sensitive strain; growth is completely inhibited at 2,4 DAPG concentrations of less than $50 \mu \mathrm{g} / \mathrm{ml}$. Focub13 and 91426 are considered tolerant strains. Radial growth is never completely inhibited, even at 2,4-DAPG concentrations of $400 \mu \mathrm{g} / \mathrm{ml}$. 
(HPLC) analysis and photodiode array spectroscopy (Fig. 4). In the extract of zone A, two compounds were visible: peak 1, eluting at $37 \mathrm{~min}$, represents the antioxidant BHT added during extraction; peak 2, at $25.7 \mathrm{~min}$, represents 2,4-DAPG based on both retention time and spectral characteristics with absorption maxima at 270 and $330 \mathrm{~nm}$ (Fig. 4A). Based on integration of the peak area, 2,4-DAPG was present in zone A at a concentration of $427 \mu \mathrm{g} / \mathrm{ml}$. In the extract from zone B, 2,4-DAPG also was detected at concentrations ranging from 300 to $345 \mu \mathrm{g} / \mathrm{ml}$, representing, on average, $76 \%$ of the concentration detected in zone A (Fig. 4B). In the extract from zone C, 2,4-DAPG still could be detected at concentrations of 2.7 to $40 \mu \mathrm{g} / \mathrm{ml}$, representing 0.6 to $9 \%$ of the initial $2,4-$ DAPG concentration as detected in zone A (Fig. 4C). These results demonstrated that most tolerant $F$. oxysporum strains were capable of metabolizing high concentrations of 2,4DAPG.

\section{Degradation of 2,4-DAPG through deacetylation.}

The identification of 2,4-DAPG degradation intermediates may facilitate the determination of which mechanism or mechanisms are used by tolerant $F$. oxysporum strains to metabolize 2,4-DAPG. For most strains, compounds in addition to BHT (peak 1) and 2,4-DAPG (peak 2) emerged in zones B and C (Fig. 4B and C). Based on retention time and spectral analysis, peak 4 matched with fusaric acid. Peak 5 could not be characterized by photodiode array spectroscopy at first instance. Only for strain R23, an additional compound (peak 3) was detected by HPLC with a retention time of $18.5 \mathrm{~min}$ and with an absorption maximum at $285 \mathrm{~nm}$ (Fig. 5A and B). The spectral characteristics and retention time matched those of monoacetylphloroglucinol (MAPG) (Fig. 5B), suggesting that 2,4DAPG is metabolized to MAPG via deacytelation. To further verify whether strain R23 is capable of metabolizing 2,4DAPG by deacetylation, resulting in generation of MAPG and possibly phloroglucinol, accurate mass liquid chromatography-mass spectrometry (LC-MS) was performed on metabolites extracted from zones B and C. For all compounds analyzed, the observed mass was within 5 ppm of the expected mass, thus confirming the identity of 2,4-DAPG, MAPG, and fusaric acid (Table 2). Peak 5 represented a compound with a $\mathrm{m} / \mathrm{z}$ of 127.0390 and deduced elemental composition of

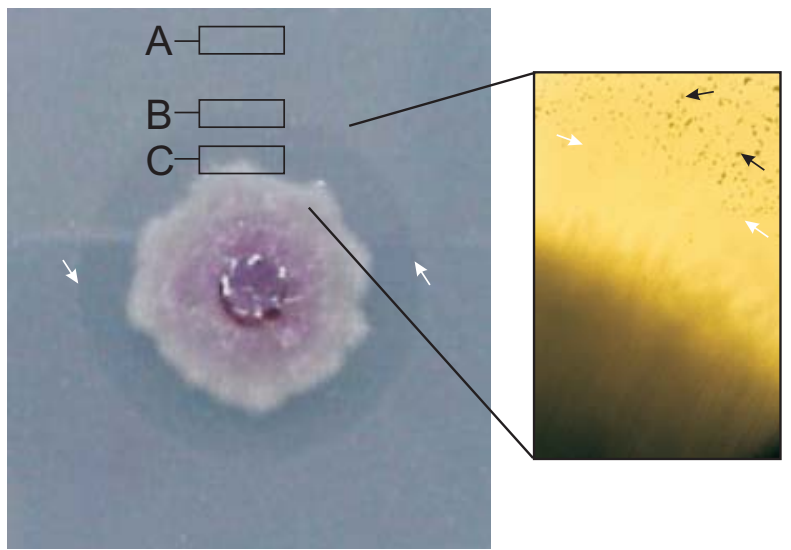

Fig. 3. Two consecutive enlargements of a plate containing 2,4-diacetylphloroglucinol (2,4-DAPG) at $400 \mu \mathrm{g} / \mathrm{ml}$ and the tolerant Fusarium oxysporum Focub13. At this concentration, 2,4-DAPG is partly precipitated (see also black particles in inset, indicated by black arrows), resulting in an opaque plate. The precipitated 2,4-DAPG disappears in the vicinity of the growing mycelium, resulting in the formation of a halo (indicated by white arrows). Indicated are the zones (A, B, and C) typically used for 2,4-DAPG concentration and metabolization analysis by methanol extraction followed by high-performance liquid chromatography analysis.
$\mathrm{C}_{6} \mathrm{H}_{6} \mathrm{O}_{3}$, which corresponds to phloroglucinol. The accumulation of phloroglucinol and MAPG could not be detected in control plates (without fungal strains) amended with 2,4DAPG (data not shown). For the other 2,4-DAPG-tolerant $F$. oxysporum strains, the intermediates MAPG and phloroglucinol could not be detected either. This may suggest another
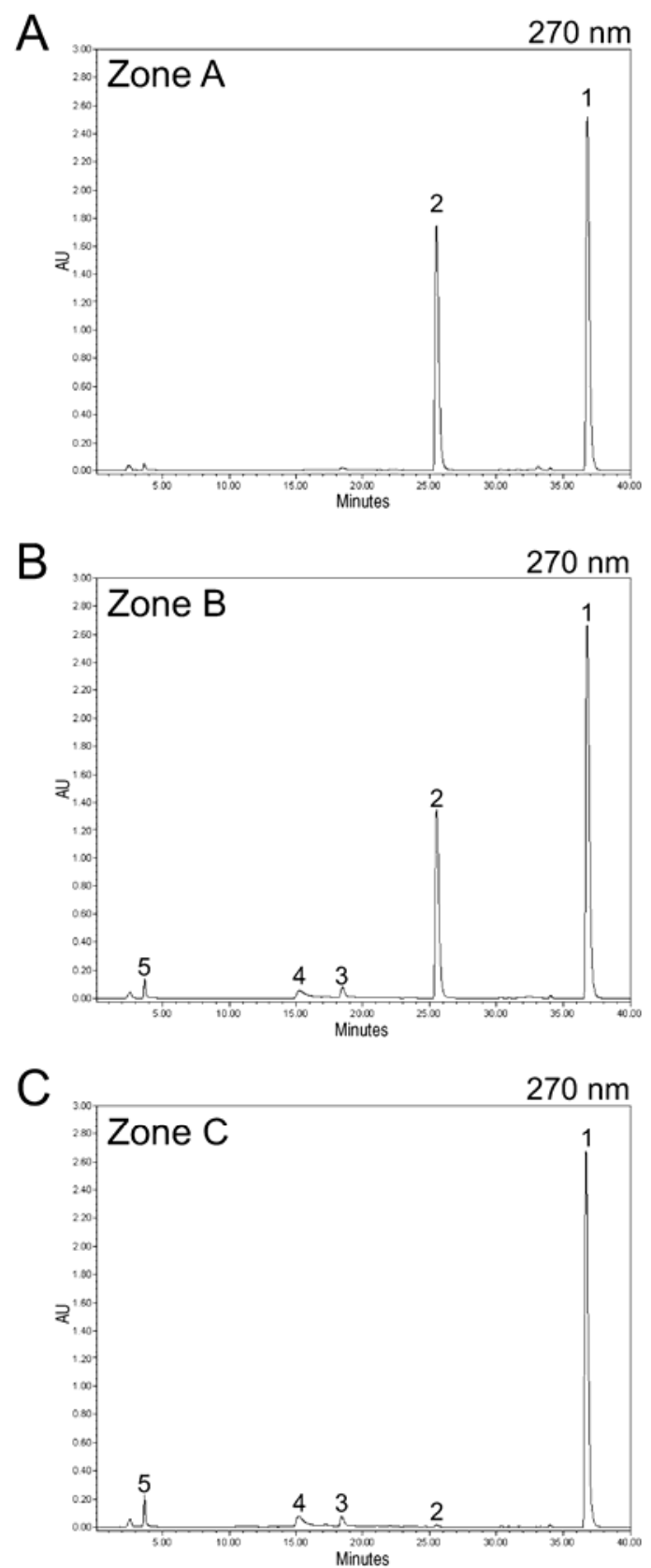

Fig. 4. Chromatogram, recorded at $270 \mathrm{~nm}$, of the methanol extract of the typical agar plate zones of 2,4-diacetylphloroglucinol (2,4-DAPG)-tolerant Fusarium oxysporum R23. In all three panels, butylated hydroxytoluene, the antioxidant present in the methanol extraction solvent, is visible (peak 1). Both the peak height and surface area underneath each peak is indicative for the concentration of each particular compound. A, Chromatogram of the opaque zone, which is indicative for the initial 2,4-DAPG concentration present in the plate (peak 2). B, Chromatogram of the zone around the halo. The height of peak 2 is reduced and other peaks emerge (peaks 3, 4, and 5). C, Chromatogram of the halo zone. Peak 2 has vanished almost completely, indicating that there is almost no 2,4-DAPG present in this zone. Peaks 3, 4, and 5 further increased in height. Based on retention time, spectral analysis and accurate mass spectrometry, peak 3 was identified as monoacetylphloroglucinol, peak 4 as fusaric acid, and peak 5 as phloroglucinol. 
pathway of 2,4-DAPG degradation or a highly efficient processing of MAPG into phloroglucinol. This latter hypothesis was supported by mass spectrometry analysis for the 2,4DAPG-tolerant strain 91426, showing trace elements of MAPG (m/z 169.0498) and the accumulation of phlorogluci$\mathrm{nol}(\mathrm{m} / \mathrm{z}$ 127.0390). Collectively, these results strongly suggest that deacetylation is, at least for some of the 2,4-DAPG-tolerant $F$. oxysporum strains, one of the initial steps in the degradation of 2,4-DAPG.

\section{Fusaric acid production varies significantly} among $F$. oxysporum strains.

As was demonstrated previously (Duffy and Défago 1997, Notz et al. 2002), fusaric acid negatively affects the biosynthesis of 2,4-DAPG in several strains of $P$. fluorescens. This
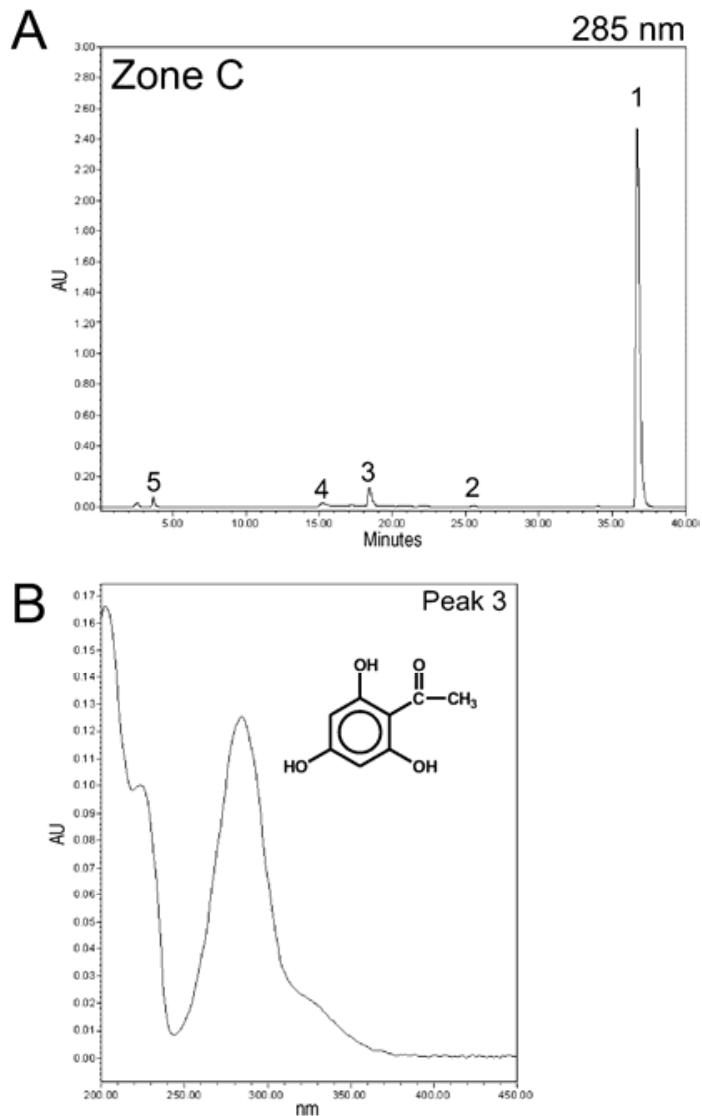

Fig. 5. A, Chromatogram, recorded at $285 \mathrm{~nm}$, of the methanol extract of the halo zone (zone C) of 2,4-diacetylphloroglucinol-tolerant Fusarium oxysporum R23. Peak 1, the antioxidant present in the methanol extraction solvent; peak 2, 2,4-DAPG concentration present in the plate; peak 3, monoacetylphloroglucinol; peak 4, fusaric acid; and peak 5, phloroglucinol B, Spectral characteristics analysis of peak 3 matches monoacetylphloroglucinol (MAPG) (see inset). finding may suggest that 2,4-DAPG-sensitive strains of $F$. oxysporum rely on the production of high fusaric acid levels to keep 2,4-DAPG accumulation in their habitat to a minimum, whereas the production of fusaric acid may not be as important for the 2,4-DAPG-tolerant $F$. oxysporum strains. Therefore, we determined the fusaric acid production of all 117 strains in an in vitro assay (Fig. 6). Among both 2,4-DAPGsensitive and -tolerant strains, the fusaric production levels varied considerably, ranging from $1,362 \mathrm{mg} / \mathrm{g}$ of biomass dry weight for Fol32 to nondetectable levels for 22 of the 117 strains. Subsequently, the effect of 2,4-DAPG on fusaric acid production in $F$. oxysporum strains was studied. Six strains were included in this assay: three 2,4-DAPG-sensitive strains varying significantly in their fusaric acid production levels (Fo47, 92T6, and Forl1) and three 2,4-DAPG-tolerant strains, also varying significantly in their fusaric acid production levels (Focub2, 91426, and Fom1127). For each strain, 28 individual liquid cultures were grown; 2,4-DAPG was added to 14 cultures at a final concentration of $25 \mu \mathrm{g} / \mathrm{ml}$, which is not lethal for all the strains tested. 2,4-DAPG concentrations were measured every $24 \mathrm{~h}$ and, if necessary, readjusted to concentrations of $25 \mu \mathrm{g} / \mathrm{ml}$. In the case of strains Fo47, 92T6, Forl1, and Fom1127, the presence of 2,4-DAPG did not result in a statistically significant reduction or enhancement of fusaric acid production levels relative to the control treatment without 2,4-DAPG. However, exposure of the tolerant strains 91426 and Focub2 to 2,4-DAPG led to opposite responses. For strain 91426, addition of 2,4-DAPG resulted in a significant increase in fusaric acid production levels from $9 \pm 0.04$ to $42 \pm 0.2 \mu \mathrm{g} / \mathrm{g}$ of biomass dry weight, seven days postinoculation; in contrast, addition of 2,4-DAPG to cultures of strain Focub2 reduced fusaric acid production more than fourfold, from $414 \pm 45$ to $92 \pm 1 \mu \mathrm{g} / \mathrm{g}$ of biomass dry weight, at 7 days postinoculation (Fig. 7). Thus, the effect of 2,4-DAPG on fusaric acid production may be strain dependent.

\section{Relationship between 2,4-DAPG tolerance and traits of $F$. oxysporum.}

2,4-DAPG tolerance of the $F$. oxysporum strains tested in this study was not linked to their pathogenicity. The percentage of tolerant strains was 22 and $14.5 \%$ among the nonpathogenic and pathogenic strains analyzed, respectively. The pathogenic 2,4-DAPG-tolerant strains corresponded to eight different formae speciales and also had various geographic origins (Table 1). Similarly, tolerant strains were found within several IGS types, which were distributed all along the dendrogram, suggesting that 2,4-DAPG-tolerant strains of F. oxysporum are genetically distant (Fig. 1). There also was no evidence of any correlation between 2,4-DAPG sensitivity or tolerance and fusaric acid production levels. The 2,4-DAPG-tolerant strains R23, Fom38, Focub2, and 92T11 produced considerable amounts of fusaric acid $(600,464,282$, and $256 \mathrm{mg} / \mathrm{g}$ of biomass dry weight, respectively), whereas Fom1127, Foa2, and

Table 2. Elemental composition and identification by mass spectrometryof the peaks detected in Figure $4^{\mathrm{a}}$

\begin{tabular}{lcccc}
\hline & & & \multicolumn{2}{c}{ Exact mass $[\mathbf{M}+\mathbf{H}]^{+}$} \\
\cline { 3 - 5 } Peak number & Compound name & Elemental composition & Calculated & \multicolumn{2}{c}{ Measured } \\
\hline 2 & $2,4-\mathrm{DAPG}$ & $\mathrm{C}_{10} \mathrm{H}_{10} \mathrm{O}_{5}$ & 211.0606 & 211.0603 \\
3 & $-\mathrm{H}_{2} \mathrm{O}$ & $\mathrm{C}_{10} \mathrm{H}_{8} \mathrm{O}_{4}$ & 193.0501 & 193.0493 \\
& $\mathrm{MAPG}$ & $\mathrm{C}_{8} \mathrm{H}_{8} \mathrm{O}_{4}$ & 169.0501 & 169.0498 \\
5 & $-\mathrm{H}_{2} \mathrm{O}$ & $\mathrm{C}_{8} \mathrm{H}_{6} \mathrm{O}_{3}$ & 151.0400 & 1.0395 \\
4 & Phloroglucinol & $\mathrm{C}_{6} \mathrm{H}_{6} \mathrm{O}_{3}$ & 127.0395 & -1.7 \\
\hline
\end{tabular}

${ }^{a}$ Measured masses were determined by accurate mass liquid chromatography-mass spectrometry on a high-resolution time-of-flight mass spectrometer with lock mass correction. 2,4-DAPG = 2,4-diacetylphloroglucinol and MAPG = monoacetylphloroglucinol. 
Foap2 produced no detectable amounts. The other 2,4-DAPGtolerant $F$. oxysporum strains produced fusaric acid at concentrations ranging from 3.6 to $161 \mathrm{mg} / \mathrm{g}$ of biomass dry weight.

\section{DISCUSSION}

Studies on interactions between antagonistic microorganisms and plant-pathogenic fungi mostly focus on a multitude of factors related to the behavior of the microbial antagonist (the antagonist perspective), i.e., how biocontrol agents affect pathogens; which mechanisms, metabolites, and genes are involved; and how antagonists behave and survive after introduction into a specific environment. In contrast, relatively few studies have focused on the 'pathogen perspective,' i.e., responses of plant pathogens to microbial antagonists (Duffy et al. 2003). The results of this study demonstrate that specific plant-pathogen populations harbor strains with adequate defense mechanisms against 2,4-DAPG, a broad-spectrum antibiotic produced by multiple strains of antagonistic P. fluorescens. Among a collection of 117 genotypically different strains of $F$. oxysporum, con- taining both saprophytic and plant-pathogenic strains, $\approx 17 \%$ were relatively tolerant to high 2,4-DAPG concentrations. These 2,4-DAPG-tolerant strains can be found at different geographical locations, within different formae speciales, and within different genetic backgrounds (IGS types). Our results showed that 2,4-DAPG tolerance did not correlate with formae speciales, geographic location, genetic background (IGS type), fusaric acid production level, and being plant pathogenic or saprophytic. These results indicate that 2,4-DAPG tolerance is distributed within and among different $F$. oxysporum populations worldwide.

Next to frequency and distribution, the level of tolerance is also of particular interest. The tolerant $F$. oxysporum strains can cope with relatively high 2,4-DAPG concentrations which are, in many cases, more than 10 times higher than the concentrations lethal to the sensitive strains. A similar level of tolerance was reported for another soil borne plant pathogen, the oomycete Pythium deliense (de Souza et al. 2003a). Pythium deliense stood out in a collection of Pythium spp., most of which had a level of sensitivity to 2,4-DAPG similar to that of
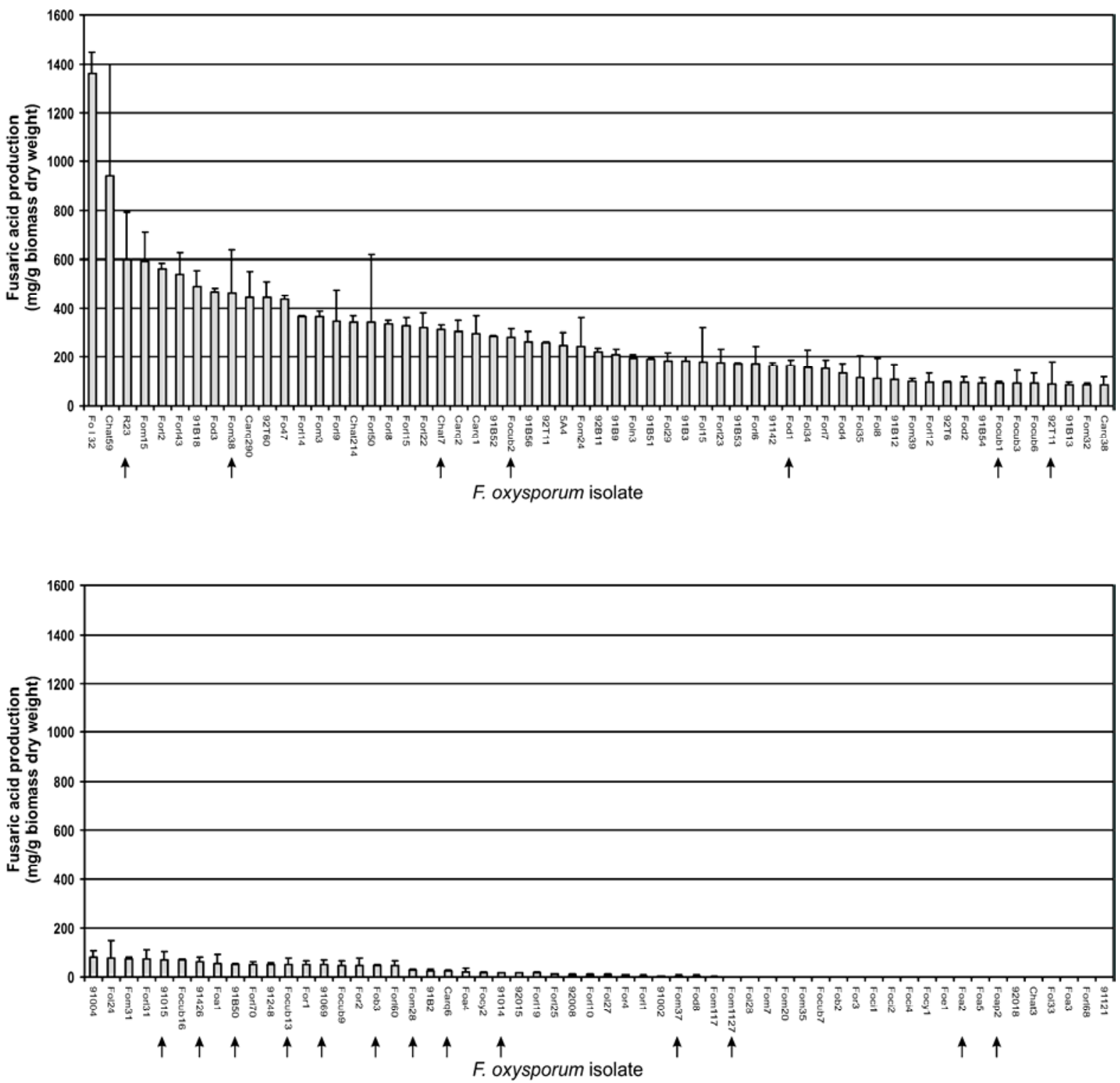

Fig. 6. Fusaric acid production by Fusarium oxysporum strains, represented as the average amount ( $\mu \mathrm{g})$ per gram of dry-biomass mycelium. Potato dextrose broth was inoculated with agar plugs containing mycelium and incubated at $25^{\circ} \mathrm{C}$ for 7 days in a rotary shaker. The amount of fusaric acid was determined by high-performance liquid chromatography analysis. The mycelium was harvested by filtration, and subsequently freeze dried and weighed. Arrows indicate the 2,4-diacetylphloroglucinol-tolerant strains and the error bars represent he standard error of the mean of two experiments. 
the sensitive $F$. oxysporum strains described in this study. Only one strain of Pythium deliense was tested; therefore, the question remains whether other Pythium deliense strains also are tolerant. In studies on the efficacy of biocontrol agents, often only one single strain of the target pathogen is tested. When considering the observed variations in sensitivity to antagonistic traits in this and other studies (Duffy et al. 2003), testing of only a few strains of a pathogen may well be inadequate for determining the value of a particular trait for the efficacy of biocontrol.

The results obtained in HPLC-PDA and LC-QTOF-MS analyses strongly indicated that deacetylation of 2,4-DAPG to MAPG and phloroglucinol is among the initial mechanisms by which tolerant $F$. oxysporum strains resist $2,4-$ DAPG. Previous studies by de Souza and associates (2003a) have clearly shown that the lower the number of acetyl groups the lower the toxicity of phloroglucinol derivatives. Although we were able to detect phloroglucinol, this compound itself probably is further metabolized by the process of ring fission as described previously for $F$. solani (Walker and Taylor 1983). We have not yet characterized the enzymes or the coding genes, which are responsible for the deactylation process. Possible candidates may be deacetylases or acetyltransferases. The latter may be of particular interest because such an enzyme, trichothecene 3-O-acetyltransferase, already was described for trichothecene detoxification in $F$. graminearum, and probably is necessary for self protection (Kimura et al. 1998a). The coding gene, Tri101, is not located within the gene cluster necessary for the trichothecene synthesis; therefore, Kimura and associates (Kimura et al. $1998 \mathrm{~b}$ ) postulated that this gene has evolved independently and was acquired by horizontal gene transfer. A homologous gene was found in F. sporotrichioides (Kimura et al. 1998b).
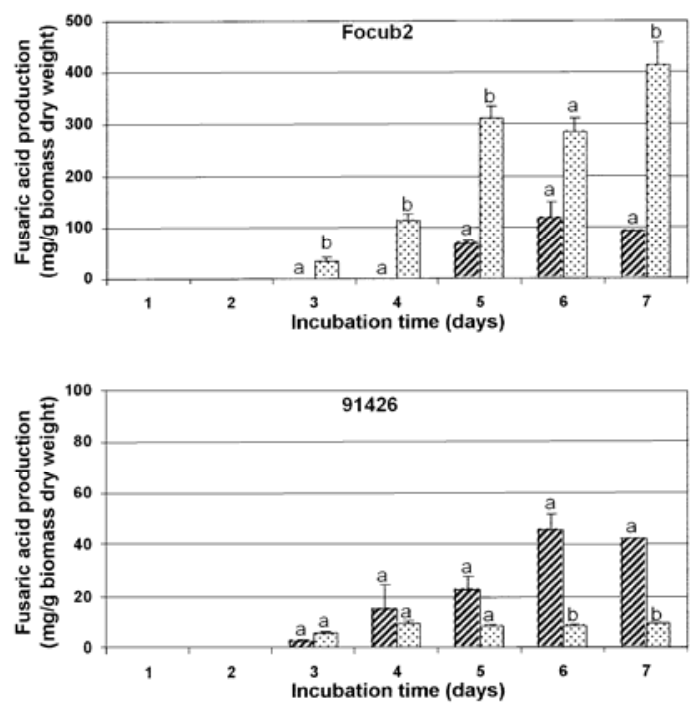

Fig. 7. Fusaric acid production by Fusarium oxysporum strains Focub2 and 91426 in the presence (shaded bars) or absence (dotted bars) of $25 \mu \mathrm{g}$ of 2,4-DAPG per ml. Fusaric acid production was measured over a period of seven days after initiation of mycelial growth and is represented as the average amount $(\mu \mathrm{g})$ per gram dry-biomass mycelium. For each strain and treatment, 28 round flasks containing potato dextrose broth were inoculated with agar plugs containing mycelium and were incubated at $25^{\circ} \mathrm{C}$ in a rotary shaker. In the case of the antibiotic treatment, the medium was supplemented with $25 \mu \mathrm{g}$ of 2,4-DAPG per ml. Every $24 \mathrm{~h}$, two flasks were harvested and analyzed for fusaric acid content, 2,4-DAPG content (when applicable), and mycelium dry weight. In case the 2,4-DAPG concentration had decreased, 2,4-DAPG was added to the remaining flask to elevate the concentration to $25 \mu \mathrm{g} / \mathrm{ml}$. The error bars represent the standard error of the mean (SEM). For each timepoint, different letters indicate a statistically significant difference $(P=0.05)$.
The strategies for coping with antibiotics from biocontrol agents appear to be as diverse and based, in part, on similar mechanisms as described for resistance to other toxins, i.e., enzymatic degradation, efflux mechanisms, and changing the target site of the toxin. Next to our example of degradation of 2,4-DAPG by different $F$. oxysporum strains, Botrytis cinerea also can degrade 2,4-DAPG by means of laccase activity (A. Schouten, G. van den Berg, and J. M. Raaijmakers, unpublished data). Two tolerant $F$. oxysporum strains, 91015 and 91B50, did not show an apparent degradation of 2,4-DAPG. Although these strains were not further studied in detail, their tolerance to 2,4-DAPG may be caused, in part, by an efflux mechanism as demonstrated previously for $B$. cinerea (Schoonbeek et al. 2002).

To obtain additional information on the defense capabilities of both sensitive and tolerant $F$. oxysporum strains, fusaric acid production levels also were determined. Fusaric acid can directly affect 2,4-DAPG biosynthesis by repressing phlA expression (Notz et al. 2002). In the present study, a significant variation in fusaric acid production levels was observed for $F$. oxysporum. These results confirm and extend results obtained by Bacon and associates (1996), who analyzed 78 strains belonging to different Fusarium spp. The results in this study showed that there is a significant percentage of $F$. oxysporum strains that does not produce detectable levels of fusaric acid under the tested conditions. Other strains were capable of producing relatively high amounts of fusaric acid. These levels were not correlated with formae speciales, genetic background, and, in particular, 2,4-DAPG tolerance. The different tolerant strains produced various amounts of fusaric acid. For three of those, Fom1127, Foa2, and Foap2, no fusaric acid production was detected, whereas four strains, R23, Fom38, Focub2, and 92T11, produced relatively high amounts. Thus, plant-pathogenic strains Fom 38 and Focub2 would be particularly difficult to control by 2,4-DAPG-producing biocontrol agents. Furthermore, one may postulate that the presence of sublethal concentrations of 2,4-DAPG may trigger fusaric acid production in $F$. oxysporum strains to repress 2,4-DAPG synthesis in Pseudomonas fluorescens to prevent further accumulation of 2,4-DAPG in their habitat to toxic concentrations. The results of this study further showed that the effect of 2,4-DAPG on fusaric acid production in $F$. oxysporum is strain dependent. Interestingly, fusaric-acid-mediated repression of 2,4-DAPG synthesis in Pseudomonas spp. also was shown to be strain dependent: fusaric acid blocked 2,4-DAPG biosynthesis in $P$. fluorescens CHA0 but not in strain Q2-87 (Duffy et al. 2004). In this context, Duffy and associates (2004) suggested that sensitivity of 2,4-DAPG-producing Pseudomonas strains to fusaric acid partly explains the variation in biocontrol efficacy. Given that 2,4DAPG production in Pseudomonas spp. may vary depending on abiotic or biotic conditions, it would be interesting to determine whether there are differences with respect to fusaric acid production between the sensitive and tolerant $F$. oxysporum isolates when exposed to different 2,4-DAPG concentrations. The results of the present study, showing considerable variation in sensitivity of $F$. oxysporum strains to 2,4-DAPG and the ability of multiple strains to degrade 2,4-DAPG, provide a new addition to the multitude of biotic factors that determine the efficacy of biological control with antagonistic Pseudomonas strains.

The result on fusaric acid production capabilities may lead to an additional conclusion, which is not within the scope of this article but nevertheless is noteworthy. Because there were enormous variations in fusaric acid production levels within all the formae speciales, one may postulate that fusaric acid is not a virulence factor, as suggested before (Bacon et al. 1996; Bryden et al. 2001). Several pathogenic strains, which did not produce detectable amounts of fusaric acid under the tested conditions, 
are known to be virulent and produce symptoms on their host plant. Conversely, the saprophytic strain Fo47, a strain which can colonize plants without showing any symptoms and which frequently is used in the biocontrol of pathogenic $F$. oxysporum strains (de Souza et al. 2003a; Duijff et al. 1998; Fravel et al. 2003), has high fusaric acid production capability. Similar results were reported for F. moniliforme (Bacon et al. 1996). Therefore, the role of fusaric acid in pathogenicity remains unresolved. In this respect, Duffy and associates (2004) speculated that the broad-spectrum antimicrobial activity of fusaric acid and other mycotoxins may be of potential importance for survival of the producing strains in crops and crop residues.

Handelsman and Stabb (1996) suggested that most biocontrol agents suppress disease via more than one mechanism, and resistance to multiple antagonistic traits should occur only at a very low frequency. Second, antagonistic microorganisms are thought to operate in microsites on the plant surface where only a fraction of the pathogen population is exposed during a short period of its life cycle. Additionally, Sevéno and associates (2002) suggested that, in case of antibiosis, only minute amounts of the compounds are produced by the biocontrol agent as opposed to the inundative application of chemical pesticides. These conditions are, among others, expected to exert a relatively low selection pressure for the buildup of resistance in pathogen populations to biocontrol. Overall, our results emphasize again that this view is based merely on the initially limited amount of experimental data and that plant pathogens probably already have developed ample and effective defense strategies against biocontrol agents (Duffy et al. 2003). Consequently, as found for any other control measurement, one may postulate that the incidence of insensitive plant pathogens will increase with the increasing use of biocontrol agents. Preliminary results of recent experiments performed in collaboration with P. A. H. M. Bakker and M. Viebahn (Utrecht University, The Netherlands) suggest that this is indeed happening. In a field trial, in which the survival of genetically modified $P$. fluorescens strains was monitored during successive wheat cultivation for several years, introduction of a transgenic Pseudomonas strain producing 2,4-DAPG caused transient shifts in indigenous Fusarium populations toward strains that efficiently degrade $2,4-\mathrm{DAPG}$. Thus, it becomes increasingly evident that biocontrol strategies have to anticipate the fact that plant pathogen populations are not static but, rather, dynamic entities, capable of rapidly adapting to and coping with adverse conditions.

\section{MATERIALS AND METHODS}

\section{Fusarium oxysporum strains, growth, and storage conditions.}

In all, 117 strains of $F$. oxysporum were analyzed (Table 1). The collection included 76 pathogenic strains representative of 13 different formae speciales, and 41 strains representative of the genetic diversity found within soil borne populations of different soils (Edel et al. 1997, 2001). The 29 strains with the first two digits 91 or 92 were nonpathogenic on flax, melon, and tomato (Steinberg et al. 1997). The other soil borne strains were isolated from samples collected from cultivated fields with various vegetable crops, including melon and tomato, in which no Fusarium diseases were observed. Thus, the soil borne strains included in this study were considered to be saprophytic strains.

F. oxysporum strains were grown at $25^{\circ} \mathrm{C}$ on potato dextrose agar (PDA; Oxoid Ltd., Basingstoke, England) plates by inoculation with conidia from frozen $\left(-80^{\circ} \mathrm{C}\right)$ stock solutions. To preserve $F$. oxysporum strains, conidia were collected from the sporulating cultures 7 to 14 days after inoculation by scraping and using $2 \mathrm{ml}$ of potato dextrose broth. This suspension was mixed with an equal volume of $50 \%$ (vol/vol) sterilized glycerol, dispensed in $1 \mathrm{ml}$ aliquots, and stored at $-80^{\circ} \mathrm{C}$.

\section{Genetic characterization \\ of the plant-pathogenic $F$. oxysporum strains.}

A subsample of 32 pathogenic strains was characterized by restriction fragment length polymorphism (RFLP) analysis of the ribosomal IGS as described previously (Edel et al. 1995, 1997). A fragment of the IGS was amplified by polymerase chain reaction with oligonucleotide primers PNFo (5'CCCGCCTGGCTGCGTCCGACTC-3') and PN22 (5'-CAAG CATATGACTACTGGC-3') and digested with seven restriction enzymes: AluI, HaeIII, HinfI, MspI, RsaI, ScrFI, and XhoI. Each strain was assigned to an IGS type defined by the combination of the restriction patterns obtained with the seven enzymes. In addition, the IGS types corresponding to 40 nonpathogenic strains were determined previously (Edel et al. 1997, 2001). The pairwise restriction site differences between IGS types were represented as a dendrogram with the computer program NTSYS (numerical taxonomy and multivariate analysis system, version 2.0, State University of New York, Stony Brook, U.S.A.).

\section{2,4-DAPG sensitivity assay.}

Plates containing one-fifth-strength potato dextrose (Oxoid), supplemented with 1.5 mM McIlvaine's citric acid-phosphate buffer (Sober 1970) set at $\mathrm{pH} 6.0$, and $1.5 \%$ (wt/vol) agar (Oxoid) were supplemented with HPLC-purified 2,4-DAPG (de Souza et al. 2003a) at 0 (control), 25, 50, 100, 200, or 400 $\mu \mathrm{g} / \mathrm{ml}$, and inoculated with a plug ( $5 \mathrm{~mm}$ in diameter) of mycelium. To properly dissolve 2,4-DAPG, $100 \times$ concentrated stock solutions were prepared in ethanol and added to liquid agar medium $\left(50^{\circ} \mathrm{C}\right)$ immediately prior to pouring. An equal amount of ethanol was added to the control. After incubation for 5 days at $25^{\circ} \mathrm{C}$, the radial growth was measured. For each 2,4-DAPG concentration, two replicates were used. The experiment was performed twice.

\section{Fusaric acid production by $\boldsymbol{F}$. oxysporum strains.}

To determine the fusaric acid production capabilities of the F. oxysporum strains, the method described by Notz and associates (2002) was used with modifications. Liquid Czapek Dox (Oxoid) medium, supplemented with $1.5 \mathrm{mM}$ Mcllvaine's citric acid-phosphate buffer ( $\mathrm{pH} 6.0$ ), was prepared according to the manufacturer's instructions and dispensed in 100-ml aliquots in flat-bottom flasks. After sterilization, the medium was inoculated with three PDA plugs containing mycelium and incubated for 7 days at $24^{\circ} \mathrm{C}$ in a rotary shaker at $180 \mathrm{rpm}$. The mycelium was harvested by filtration over Miracloth (Calbiochem, San Diego, CA, U.S.A.) and the fungal biomass was determined after freeze-drying. The total volume of the culture filtrate was measured and the fusaric acid concentration was calculated by measuring the area under the fusaric acid peak detected at $270 \mathrm{~nm}$ by the array detector after HPLC. Different concentrations of purified fusaric acid (Sigma-Aldrich, St. Louis) were used to calculate the relation between peak area and concentration.

The effect of 2,4-DAPG on the fusaric acid accumulation was determined by inoculating 28 flat-bottom flasks containing $100 \mathrm{ml}$ of Czapek Dox medium, supplemented with 1.5 $\mathrm{mM}$ McIlvaine's citric acid-phosphate buffer set at $\mathrm{pH}$ 6.0, with mycelium from a $F$. oxysporum strain. 2,4-DAPG was added to 14 of these cultures to a final concentration of 25 $\mu \mathrm{g} / \mathrm{ml}$ at the time of inoculation. For 7 days, four flasks were sampled every $24 \mathrm{~h}$, two with and two without 2,4-DAPG. The mycelium was harvested by filtration over Miracloth (Calbio- 
chem) and the fungal biomass was determined after freezedrying. The total volume of the culture filtrate was measured and both the average 2,4-DAPG (when applicable) and the fusaric acid concentrations were calculated by measuring the area under the 2,4-DAPG and fusaric acid peaks, respectively, both detected at $270 \mathrm{~nm}$ by the array detector after HPLC. When necessary, 2,4-DAPG was added to the remaining 2,4DAPG-containing cultures in order to maintain the antibiotic concentration at $25 \mu \mathrm{g} / \mathrm{ml}$ throughout the incubation period. Differences in fusaric acid production levels by Fusarium isolates exposed or not to 2,4-DAPG were analyzed statistically by a $t$ test $(P=0.05)$.

\section{HPLC-photodiode array analysis.}

2,4-DAPG was quantified by HPLC analysis on a Waters HPLC system using a Symmetry C18 reversed phase column (150 by $3.9 \mathrm{~mm}, 5 \mu \mathrm{m}$ ), preceded by a Symmetry C18 reversed-phase guard column ( 20 by $3.9 \mathrm{~mm}, 5 \mu \mathrm{m})$, which were connected to a $600 \mathrm{~S}$ system controller, 616 pump unit, 996 photodiode array detector, and $717 \mathrm{~S}$ autosampler.

From the plates containing 2,4-DAPG at $400 \mu \mathrm{g} / \mathrm{ml}, 0.1$ to 1 $\mathrm{g}$ of agar was extracted with two volumes (wt/vol) of methanol, supplemented with $0.75 \%$ (wt/vol) butylated hydroxytoluene (BHT; Sigma-Aldrich), by overnight incubation. From the methanol fraction, 10 to $50 \mu \mathrm{l}$ was injected onto the column, which had been equilibrated with $10 \%$ (vol/vol) acetonitrile and $0.1 \%$ (vol/vol) trifluoroacetic acid (TFA) (solvent A), and eluted at a flow rate of $0.5 \mathrm{ml} / \mathrm{min}$ using an isocratic flow of $100 \%$ solvent A for 2 min, a linear gradient from $100 \%$ solvent A to $100 \%$ solvent $\mathrm{B}(90 \%$ [vol/vol] acetonitrile and $0.1 \%$ [vol/vol] TFA) in $23 \mathrm{~min}$ and an isocratic flow of $100 \%$ solvent $\mathrm{B}$ for $5 \mathrm{~min}$. Under these conditions, the 2,4-DAPG standard eluted at a retention time of $25.7 \mathrm{~min}$ with an absorption maximum at $270 \mathrm{~nm}$.

The simultaneous detection and quantification of 2,4-DAPG and fusaric acid in liquid cultures was done on the same Waters HPLC system as mentioned above, but lacking the guard column. A culture filtrate sample was centrifuged at 14,000 $\times g$ for $10 \mathrm{~min}$ to remove fungal debris. An equal volume of methanol was added to the supernatant. Of this mixture, 10 to $50 \mu \mathrm{l}$ was injected onto the reverse phase column, which was equilibrated with $25 \%$ (vol/vol) methanol and $0.03 \%$ (vol $/ \mathrm{vol}$ ) TFA (solvent $\mathrm{C}$ ), and eluted by a flow rate of $1 \mathrm{ml} / \mathrm{min}$ using an isocratic flow of $100 \%$ solvent $\mathrm{C}$ for $2 \mathrm{~min}$, a linear gradient from $100 \%$ solvent $\mathrm{C}$ to $100 \%$ solvent $\mathrm{D}$ (methanol and $0.3 \%$ [vol/vol] TFA) in $13 \mathrm{~min}$, and an isocratic flow of $100 \%$ solvent $\mathrm{D}$ for $5 \mathrm{~min}$. Under these conditions, fusaric acid (SigmaAldrich) and 2,4-DAPG standards were detected at $270 \mathrm{~nm}$ (optimal wavelength) and at retention times of 7.9 and 16.2 min, respectively.

\section{LC-MS analysis.}

To identify 2,4-DAPG derivatives, samples were subjected to accurate-mass LC-MS on a high-resolution time-of-flight mass spectrometer with lock mass correction (Wolff et al. 2001). Samples were injected and separated using a Waters Alliance 2795 HT system equipped with a Luna C18-reversed phase column (150 by $2.1 \mathrm{~mm}, 3 \mu \mathrm{m}$; Phenomenex, Torrance, CA, U.S.A.) at $40^{\circ} \mathrm{C}$ and a gradient from 5 to $50 \%$ (vol $/ \mathrm{vol}$ ) acetonitril acidified with $0.1 \%$ ( vol/ $/ \mathrm{vol}$ ) formic acid at a flow rate of 0.2 $\mathrm{ml} / \mathrm{min}$. Eluting compounds were detected online at 210 to 600 $\mathrm{nm}$ by a 2996 photodiode array detector followed by a Q-TOF Ultima MS with ESI-source. The following settings were applied during the LC-MS runs: desolvation temperature of $300^{\circ} \mathrm{C}$ with a nitrogen gas flow of 500 liters/h, capillary spray at $3 \mathrm{kV}$, source temperature of $120^{\circ} \mathrm{C}$, cone at $35 \mathrm{eV}$ with nitrogen gas flow at 50 liters $/ \mathrm{h}$, collision energy at $5 \mathrm{eV}$. Positive ions in the
100 to $1,000 \mathrm{~m} / \mathrm{z}$ range were detected using a scan time of $0.9 \mathrm{~s}$ and an interscan delay of $0.1 \mathrm{~s}$. The MS was calibrated with $0.5 \%$ (vol/vol) phosphoric acid in 50\% (vol/vol) acetonitril and leucine enkaphalin was used as a lock mass. Masslynx software version 4.0 was used to control all instruments and to calculate accurate masses.

\section{ACKNOWLEDGMENTS}

The work presented here was financed in part by the Royal Netherlands Academy of Arts and Sciences. Additional financial support was obtained by an NWO-Van Gogh grant (no. VGP 83-192).

\section{LITERATURE CITED}

Alexander, N. J., McCormick, S. P., and Hohn, T. M. 1999. TRI12, a trichothecene efflux pump from Fusarium sporotrichioides: Gene isolation and expression in yeast. Mol. Gen. Genet. 261:977-84.

Bacon, C. W., Porter, J. K., Norred, W. P., and Leslie, J. F. 1996. Production of fusaric acid by Fusarium species. Appl. Environ. Microbiol. 62:4039-4043.

Bryden, W. L., Logrieco, A., Abbas, H. K., Porter, J. K., Vesonder, R. F., Richard, J. L., and Cole, R. J. 2001. Other significant Fusarium mycotoxins. In: Fusarium: Paul E. Nelson Memorial Symposium. B. A. Summerell, J. F. Leslie, D. Backhouse, W. L. Bryden, and L. W. Burgess, eds. American Phytopathological Society Press, St. Paul, MN, U.S.A.

Chelkowski, J. 1989. Fusarium: Mycotoxins, taxonomy and pathogenicity. In: Topics in Secondary Metabolism, vol. 2. Elsevier, Amsterdam.

Chin-A-Woeng, T. F. C., Bloemberg, G. V., and Lugtenberg, B. J. J. 2003. Phenazines and their role in biocontrol by Pseudomonas bacteria. New Phytol. 157:503-523.

de Souza, J. T., Arnould, C., Deulvot, C., Lemanceau, P., GianinazziPearson, V., and Raaijmakers, J. M. 2003a. Effect of 2,4-diacetylphloroglucinol on Pythium: Cellular responses and variation in sensitivity among propagules and species. Phytopathology 93:966-975.

de Souza, J. T., de Boer, M., de Waard, P., van Beek, T. A., and Raaijmakers, J. M. 2003b. Biochemical, genetic, and zoosporicidal properties of cyclic lipopeptide surfactants produced by Pseudomonas fluorescens. Appl. Environ. Microbiol. 69:7161-7172.

de Souza, J. T., Weller, D. M., and Raaijmakers, J. M. 2003c. Frequency, diversity, and activity of 2,4-diacetylphloroglucinol-producing fluorescent Pseudomonas spp. in Dutch take-all decline soils. Phytopathology 93:54-63.

De Waard, M. A. 1997. Significance of ABC transporters in fungicide sensitivity and resistance. Pestic. Sci. 51:271-275.

Duffy, B., Keel, C., and Defago, G. 2004. Potential role of pathogen signaling in multitrophic plant-microbe interactions involved in disease protection. Appl. Environ. Microbiol. 70:1836-1842.

Duffy, B., Schouten, A., and Raaijmakers, J. M. 2003. Pathogen selfdefense: Mechanisms to counteract microbial antagonism. Annu. Rev. Phytopathol. 41:501-538.

Duffy, B. K., and Défago, G. 1997. Zinc improves biocontrol of Fusarium crown and root rot of tomato by Pseudomonas fluorescens and represses the production of pathogen metabolites inhibitory to bacterial antibiotic biosynthesis. Phytopathology 87:1250-1257.

Duijff, B. J., Pouhair, D., Olivain, C., Alabouvette, C., and Lemanceau, P. 1998. Implication of systemic induced resistance in the suppression of Fusarium wilt of tomato by Pseudomonas fluorescens WCS417r and by nonpathogenic Fusarium oxysporum Fo47. Eur. J. Plant Pathol. 104:903-910.

Edel, V., Steinberg, C., Avelange, I., Laguerre, G., and Alabouvette, C. 1995. Comparison of 3 molecular methods for the characterization of Fusarium oxysporum strains. Phytopathology 85:579-585.

Edel, V., Steinberg, C., Gautheron, N., and Alabouvette, C. 1997. Populations of nonpathogenic Fusarium oxysporum associated with roots of four plant species compared to soilborne populations. Phytopathology 87:693-697.

Edel, V., Steinberg, C., Gautheron, N., Recorbet, G., and Alabouvette, C. 2001. Genetic diversity of Fusarium oxysporum populations isolated from different soils in France. FEMS (Fed. Eur. Microbiol. Soc.) Microbiol. Ecol. 36:61-71.

Fleissner, A., Sopalla, C., and Weltring, K. M. 2002. An ATP-binding cassette multidrug-resistance transporter is necessary for tolerance of $\mathrm{Gib}$ berella pulicaris to phytoalexins and virulence on potato tubers. Mol. Plant-Microbe Interact. 15:102-108.

Fravel, D., Olivain, C., and Alabouvette, C. 2003. Fusarium oxysporum and its biocontrol. New Phytol. 157:493-502. 
Gupta, A. K., Baran, R., and Summerbell, R. C. 2000. Fusarium infections of the skin. Curr. Opin. Infect. Dis. 13:121-128.

Handelsman, J., and Stabb, E. V. 1996. Biocontrol of soilborne plant pathogens. Plant Cell 8:1855-1869.

Joffe, A. Z. 1986. Fusarium Species: Their Biology and Toxicology. Wiley, New York.

Kamei, Y., and Isnansetyo, A. 2003. Lysis of methicillin-resistant Staphylococcus aureus by 2,4-diacetylphloroglucinol produced by Pseudomonas sp. AMSN isolated from a marine alga. Int. J. Antimicrob. Agents 21:71-4.

Keel, C., Weller, D. M., Natsch, A., Défago, G., Cook, R. J., and Thomashow, L. S. 1996. Conservation of the 2,4-diacetylphloroglucinol biosynthesis locus among fluorescent Pseudomonas strains from diverse geographic locations. Appl. Environ. Microbiol. 62:552-563.

Kimura, M., Kaneko, I., Komiyama, M., Takatsuki, A., Koshino, H., Yoneyama, K., and Yamaguchi, I. 1998a. Trichothecene 3-O-acetyltransferase protects both the producing organism and transformed yeast from related mycotoxins. Cloning and characterization of Tri101. J. Biol. Chem. 273:1654-61.

Kimura, M., Matsumoto, G., Shingu, Y., Yoneyama, K., and Yamaguchi, I. 1998b. The mystery of the trichothecene 3-O-acetyltransferase gene. Analysis of the region around Tri101 and characterization of its homologue from Fusarium sporotrichioides. FEBS (Fed. Eur. Biol. Soc.) Lett. 435:163-8.

Lagopodi, A. L., Ram, A. F. J., Lamers, G. E. M., Punt, P. J., Van den Hondel, Camjj, Lugtenberg, B. J. J., and Bloemberg, G. V. 2002. Novel aspects of tomato root colonization and infection by Fusarium oxysporum $\mathrm{f}$. $\mathrm{sp}$ radicis-lycopersici revealed by confocal laser scanning microscopic analysis using the green fluorescent protein as a marker. Mol. Plant-Microbe Interact. 15:172-179.

Lorie, G., Edel-Hermann, V., Gautheron, N., and Alabouvette, C. 2004 Genetic diversity of pathogenic and nonpathogenic populations of Fusarium oxysporum isolated from carnation fields in Argentina. Phytopathology 94:661-668.

Mazzola, M., Fujimoto, D. K., Thomashow, L. S., and Cook, R. J. 1995. Variation in sensitivity of Gaeumannomyces graminis to antibiotics produced by fluorescent Pseudomonas spp. and effect on biological control of take-all of wheat. Appl. Environ. Microbiol. 61:2554-2559.

Morrissey, J. P., and Osbourn, A. E. 1999. Fungal resistance to plant antibiotics as a mechanism of pathogenesis. Microbiol. Mol. Biol. Rev. 63:708-24.

Nielsen, T. H., Sorensen, D., Tobiasen, C., Andersen, J. B., Christophersen, C., Givskov, M., and Sorensen, J. 2002. Antibiotic and biosurfactant properties of cyclic lipopeptides produced by fluorescent Pseudomonas spp. from the sugar beet rhizosphere. Appl. Environ. Microbiol. 68:3416-3423

Notz, R., Maurhofer, M., Dubach, H., Haas, D., and Défago, G. 2002.
Fusaric acid-producing strains of Fusarium oxysporum alter 2,4-diacetylphloroglucinol biosynthetic gene expression in Pseudomonas fluorescens $\mathrm{CHA} 0$ in vitro and in the rhizosphere of wheat. Appl. Environ. Microbiol. 68:2229-2235.

Raaijmakers, J. M., Vlami, M., and de Souza, J. T. 2002. Antibiotic production by bacterial biocontrol agents. Antoinne Leeuwenh. 81:537547.

Schnider-Keel, U., Seematter, A., Maurhofer, M., Blumer, C., Duffy, B. Gigot Bonnefoy, C., Reimmann, C., Notz, R., Défago, G., Haas, D., and Keel, C. 2000. Autoinduction of 2,4-diacetylphloroglucinol biosynthesis in the biocontrol agent Pseudomonas fluorescens $\mathrm{CHA} 0$ and repression by the bacterial metabolites salicylate and pyoluteorin. J. Bacteriol. 182:1215-1225.

Schoonbeek, H. J., Raaijmakers, J. M., and De Waard, M. A. 2002. Fungal $\mathrm{ABC}$ transporters and microbial interactions in natural environments. Mol. Plant-Microbe Interact. 15:1165-1172.

Séveno, N. A., Kallifidas, D., Smalla, K., van, Elsas J. D., Collard, J. M., Karagouni, A. D., and Wellington, E. M. H. 2002. Occurrence and reservoirs of antibiotic resistance genes in the environment. Rev. Med. Microbiol. 13:15-27.

Sober, H. A., ed. 1970. Handbook of Biochemistry. 2nd ed. The Chemical Rubber Co., Cleveland.

Steffens, J. J., Pell, E. J., and Tien, M. 1996. Mechanisms of fungicide resistance in phytopathogenic fungi. Curr. Opin. Biotechnol. 7:348-55.

Steinberg, C., Edel, V., Gautheron, N., Abadie, C., Vallaeys, T., and Alabouvette, C. 1997. Phenotypic characterization of natural populations of Fusarium oxysporum in relation to genotypic characterization. FEMS (Fed. Eur. Microbiol. Soc.) Microbiol. Ecol. 24:73-85.

Summerbell, R. C., and Schroers, H. J. 2002. Analysis of phylogenetic relationship of Cylindrocarpon lichenicola and Acremonium falciforme to the Fusarium solani species complex and a review of similarities in the spectrum of opportunistic infections caused by these fungi. J. Clin Microbiol. 40:2866-2875.

Thomashow, L. S., and Weller, D. M. 1996. Current concepts in the use of introduced bacteria: Mechanisms and antifungal metabolites. In: PlantMicrobe Interactions. G. Stacey and N. T. Keen, eds. Chapman \& Hall, New York.

VanEtten, H., Temporini, E., and Wasmann, C. 2001. Phytoalexin (and phytoanticipin) tolerance as a virulence trait: Why is it not required by all pathogens? Physiol. Mol. Plant Pathol. 59:83-93.

Walker, J. R. L., and Taylor, B. G. 1983. Metabolism of phloroglucinol by Fusarium solani. Arch. Microbiol. 134:123-126.

Wolff, J.-C., Eckers, C., Sage, A. B., Giles, K., and Bateman, R. 2001. Accurate mass liquid chromatography/mass spectrometry on quadruple orthogonal acceleration time-of-flight mass analyzers using switching between separate sample and reference sprays. 2. Applications using the dual-electrospray ion source. Anal. Chem. 73:2605-2612. 